ACCEPTEd For publication in ApJ LetTers

Preprint typeset using IATEX style emulateapj v. 11/12/01

\title{
DETECTION OF SUPERGRANULATION ALIGNMENT IN POLAR REGIONS OF THE SUN BY HELIOSEISMOLOGY
}

\author{
Kaori Nagashima ${ }^{1}$, Junwei ZhaO ${ }^{1}$, Alexander G. Kosovichev ${ }^{1}$, and Takashi Sekit ${ }^{2}$ \\ accepted for publication in ApJ Letters
}

\begin{abstract}
We report on a new phenomenon of 'alignment' of supergranulation cells in the polar regions of the Sun. Recent high-resolution datasets obtained by the Solar Optical Telescope onboard the Hinode satellite enabled us to investigate supergranular structures in high-latitude regions of the Sun. We have carried out a local helioseismology time-distance analysis of the data, and detected acoustic travel-time variations due to the supergranular flows. The supergranulation cells in both the north and south polar regions show systematic alignment patterns in the north-south direction. The south-pole datasets obtained in a month-long Hinode campaign indicate that the supergranulation alignment property may be quite common in the polar regions. We also discuss the latitudinal dependence of the supergranulation cell sizes; the data show that the east-west cell size decreases towards higher latitudes.
\end{abstract}

Subject headings: convection - Sun: helioseismology

\section{INTRODUCTION}

Supergranules (Hart 1954; Leighton et al. 1962) are thought to be convection cells of the Sun with the size of 20-30 Mm and the lifetime of $\sim 1$ day. Although more than half a century has passed since they were found, their physical property is still puzzling and controversial. Simon \& Leighton (1964) suggested that a convective instability associated with the He ionization zone might produce the supergranular cells. Stein et al. (2007) argued that there is nothing special about the supergranulation scale except that this is the scale favored by magnetic fields interacting with convective flows. Gizon et al. (2003), Schou (2003), and Green \& Kosovichev (2007) suggested that supergranulation has properties of traveling convection waves, while Rast et al. (2004) and Lisle et al. (2004) discussed that the supergranulation patterns may be organized by giant cells. For recent discussions, see the review by Rieutord \& Rincon (2010).

Hydrodynamic processes, including supergranulation, in the polar regions of the Sun most likely play an important role in the solar dynamics and magnetic activity cycles. For example, Dikpati et al. (2010) discussed that the speed of the meridional flow in the polar regions could determine the length of the activity cycle. The processes in the polar regions, however, have not been fully investigated from the observational point of view, mainly because observing the high-latitude regions from the ecliptic plane is difficult due to severe foreshortening. Numerical simulations of the turbulent convection on the Sun have suggested some kind of latitudinal dependence of the supergranulation patterns, although convection cells of the supergranulation scale have not been clearly reproduced in the global simulations of the convection zone, except in a shallow spherical shell study (De Rosa et al. 2002). The cells resolved in the global simulations of solar convection are mostly of a giant-cell scale. According to the recent simulation results (e.g., Brun \& Toomre 2002; Miesch et al. 2006), the convective cells in the lower latitude regions may align parallel to the rotation axis. In the higher-latitude regions, the structure of the cells looks more complicated and less organized.

We take advantage of the Hinode high spatial resolution, and study the near-polar regions during the periods of the highest inclination of the solar axis to the ecliptic. Our finding from the Hinode data analysis is that the supergranulation cells in the high-latitude regions seem to align predominantly in the north-south direction. This cannot be explained by the giant-cell scale structures in the numerical simulations.

\section{OBSERVATIONS}

The Solar Optical Telescope (SOT; Tsuneta et al. 2008) onboard the Hinode satellite (Kosugi et al. 2007) has made several observing runs for local helioseismology. In particular, the polar region of the southern hemisphere was observed on 2009 March 7, and the polar region in the northern hemisphere was observed on 2009 September 25. Since the inclination of the solar rotation axis attains its maximum (about 7 degrees) in March and September every year, these are the best periods for observing the highlatitude regions. For comparison and testing, we also obtained datasets of a region close to the east limb on 2009 September 27, and a region around the disk center on 2009 December 11. All the observations were 16 hours long.

In March of 2010, SOT carried out nine helioseismology observing runs for the south polar region approximately once every three days. The observing period of each run was 12-16 hours, aside from the observation on March 16, which was 6.7 hours long.

The data acquired with a cadence of 60 seconds were Ca II H line intensity maps as well as Fe I $557.6 \mathrm{~nm}$ intensity maps. Since the field of view of the Fe-line maps obtained by the Narrowband Filter Imager (NFI) of the SOT is wider than that of the Ca-line maps obtained by the Broadband Filter Imager (BFI), we set up the telescope pointing in such a way that the solar limb is in the NFI field of view. The NFI data were then used for tracking the limb position and for determining the coordinates

${ }^{1}$ W.W. Hansen Experimental Physics Laboratory, 452 Lomita Mall, Stanford University, Stanford, CA 94305-4085, USA; kaorin@stanford.edu

2 National Astronomical Observatory of Japan, 2-21-1 Osawa, Mitaka, Tokyo 181-8588, Japan. 
on the Sun in the BFI images. For the helioseismology analysis we used the Ca II $\mathrm{H}$ data series.

\section{DATA ANALYSIS}

We first aligned the images and determined the heliographic coordinates of each image. Then, we chose several points on the Sun within the SOT field of view, as the central points for the Postel's projection (see below). We tracked the central points with the local differential rotation rate (Snodgrass 1984), and remapped each image into the heliographic coordinates by using the Postel's projection. Using the tracked and remapped images, we carried out the time-distance analysis (Duvall et al. 1997; Kosovichev \& Duvall 1997) to measure variations of acoustic travel times and detect subsurface flow structures.

The SOT observations of the regions close to the limb were carried out with fixed telescope pointings. Although the image stabilization system, the correlation tracker (CT; Shimizu et al. 2008), was turned on, the field of view was gradually drifting toward the disk center mainly due to the limb darkening. Therefore, we aligned the images by ensuring that the limb stays in the same position in the field of view, and then determined the solar coordinates of each image.

When we observe regions near the solar limb, foreshortening is significant, and appropriate remapping is required for the data analysis. We used the Postel's azimuthal equidistant projection (e.g., Bogart et al. 1995; Zaatri et al. 2008). The projected map is what we would observe by looking directly from above the region. For the dataset taken at the disk center, however, we did not project the images, because the distortion effect is negligible there. Also, since for the disk center dataset the SOT observed the region tracking its center with the rotation rate of the Sun, we did not apply any further tracking.

For the time-distance analysis, cross-correlation functions of the surface wavefield of the 5-min oscillations were calculated from the tracked and projected datasets. We measured the acoustic (phase) travel times for several distances by fitting a Gabor-type wavelet to the crosscorrelation functions (Kosovichev \& Duvall 1997). For each distance $\Delta$, we averaged the oscillation signals over an annulus of the radius $\Delta$ around a target point, and then cross-correlated the averaged signals with the oscillation signal observed at the target point. We obtained outward (from the target to the annulus) and inward (from the annulus to the target) travel times at each point in the field of view for a set of travel distances $\Delta$. The difference of the outward and inward travel times indicates the diverging/converging flow around the target point in the region of wave propagation below the surface. Note that for improving the signal-to-noise ratio we applied phase-speed filters following Duvall et al. (1997).

\section{RESULTS}

\subsection{Travel-time maps}

The outward-inward travel-time difference maps of the north polar region are illustrated in Figure 1. Panels (a), (b), and (c) show the travel times for the distances, $\Delta$, of $8.4,14.4$, and $21.1 \mathrm{Mm}$, (or $0.7^{\circ}, 1.2^{\circ}$, and $1.7^{\circ}$ ) respectively. The black regions, where the outward travel times are shorter than the inward travel times, indicate diverging flows, while the white regions correspond to converging flows. The dark cells with white boundaries are supergranular cells. In the maps for the largest annuli (panel c), the supergranular patterns are rather faint. Judging from the penetrating depths of the acoustic rays, this indicates that the supergranules are not much deeper than, say, $5 \mathrm{Mm}$, which is consistent with other helioseismology results (e.g., Woodard 2007; Sekii et al. 2007). Hereafter, we use only the travel times for $\Delta=14.4 \mathrm{Mm}$, because these traveltime maps (panel b) show the supergranular patterns most clearly.

Figure1d shows a 4-hour averaged intensity image in the $\mathrm{Ca}$ II $\mathrm{H}$ line of the north polar region. It is well-known that supergranulation is seen as a bright network structure in the chromosphere because magnetic field is concentrated at the supergranulation boundaries. In this chromospheric image the network brightenings, though faint, are seen, and their distribution is consistent with the supergranular boundaries detected in the outward-inward travel-time maps (panels a to c).

In Figure 1, the outward-inward travel-time difference maps of the south polar region (panel e), a region near the east limb (panel f), and a region around the disk center (panel g) are shown. Figure 2 shows the nine outwardinward travel-time difference maps of the south pole obtained for each of the nine observing runs in March of 2010. In these maps, the supergranular patterns in both polar regions and the east limb region are seen as clearly as in the travel time map of the disk center. In the high-latitude region, however, the cells seem to align in the direction roughly from north to south, with some tilt. The 'alignment' patterns exist in both of the north and south polar regions; Figure 2 suggests that the 'alignment' is probably a characteristic structure in the polar regions. There also seem to be areas with weak diverging flows between the aligned cells. The alignment seems to be coherent for a distance of 2-3 cells.

\subsection{Correlation of the travel-time difference maps}

For a quantitative characterization of the alignment, we calculated two-dimensional correlations of the travel-time difference maps. Figure 3 shows the correlation functions of the travel-time difference maps for the north polar region and the lower-latitude regions as well. The correlation function of the north polar datasets (panel d) does indicate a striking alignment in the map, i.e., the correlation is the strongest in the northeast-southwest direction. On the other hand, for the disk center and the east limb region maps (panels e and f) the correlation shows no particular preference in direction; namely, it shows a nearly random distribution.

Actually, the strength of the 'alignment' differs from dataset to dataset. The alignment, however, does exist in the averaged correlation functions of the travel-time difference maps, calculated for different latitudes (Figure 4). The alignment is most notable at the highest latitude, as shown in the bottom panel. The alignment is almost in the north-south direction, but not precisely. We estimated the mean direction and its variance for the nine datasets. We measured the peak position angle along a $20-\mathrm{Mm}$ circle centered at the origin of the correlation maps, and 
found that the mean and the variance alignment angles are $90.1 \pm 37.0$ degrees at $72^{\circ}$ in latitude, $102.5 \pm 23.2$ degrees at $76^{\circ}$, and $92.1 \pm 11.5$ degrees at $83^{\circ}$. Note that here the direction of 0 degree is to the west, and 90 degrees is to the north. This analysis indicates: 1) the alignment is, on average, in the nearly north-south direction, and 2) the variance of the alignment angle is smaller in the higher latitude regions. On a short time scale, the alignment might be affected by the polar dynamics, such as varying differential rotation (Ye \& Livingston 1998), meridional flow, and a polar vortex (e.g., Gilman 1979).

The correlation functions of the travel-time maps also show us variation of the cell size with latitude. We have found that the east-west size of the supergranular cells in the high-latitude regions tends to be smaller than in the low-latitude regions. We define the cell radius (a half of the full size) as a correlation length: the distance in $x$ (east-west) direction from the origin to the point where the correlation is down to zero in the correlation map. The cell correlation radii are 10-12 Mm in the north/south polar regions, and 13-14 Mm in the east limb region and around the disk center. Moreover, from the average of the correlation function over the nine south polar datasets, we obtained the cell radii as $12.0 \pm 0.4 \mathrm{Mm}$ around $72^{\circ}$ in latitude, $11.8 \pm 0.2 \mathrm{Mm}$ around $76^{\circ}$, and $11.2 \pm 0.2 \mathrm{Mm}$ around $83^{\circ}$. The errors are estimated from the variance of the nine datasets. This implies that the cell size depends on latitude. This type of latitudinal dependence is consistent with the results obtained from f-mode helioseismology analysis by Hirzberger et al. (2008) and from Ca II K line observations of the chromospheric network (Brune \& Wöhl 1982; Münzer et al.(1989)Münzer, Hanslmeier Schröter), although these observations were for mid- or lower-latitude regions. Note that by this method we could not define the cell size in the north-south direction because the correlation function does not drop to zero in this direction (see Figure 4). This might be related to the fact that regions with weak diverging flows are located between the aligned cells in the north-south direction as can be seen in the travel-time maps (Figures 1 and 2), but this suggestion needs further investigation.

We verified that the alignment is not due to some kind of artifacts caused by the limb observation, because the alignment is not found in the control study of the east limb region. In general, any kind of limb-observation effect would not affect positions of the cells in the travel-time maps, thereby producing a spurious alignment. Also, it is unlikely that projection effects affected our estimates of the cell size in the east-west direction.

\subsection{Temporal evolution}

The upper panel of Figure [5] shows the combined outward-inward travel-time difference map of the nine south polar datasets of March of 2010. This is a view from above the South Pole of the Sun. The first observation of March 3 is the rightmost one, and the following observations are located sequentially counter-clockwise. We calculated the longitudinal difference between the observing dates by using the differential rotation rate (Snodgrass 1984) at the central latitude of each observation. Note that the supergranulation pattern in Figure 5 looks fainter than in the individual maps (Figure 2) because of overlapping of the different time intervals.

The lower panels show the last two datasets, the temporal separation between which was only 15.5 hours, and their fields of view significantly overlapped. Many features in the overlapping region look similar; a clear example is along the line at angle of 240 degrees. Obviously, the cells and their alignment pattern survive at least 15.5 hours, which is consistent with the known fact that the supergranular cell lifetime is about a day. For a detailed study of the temporal evolution we need more consecutive datasets.

\section{DISCUSSIONS}

We have found evidence indicating that: 1) supergranular cells tend to align roughly in the north-south direction (with some tilt) in the polar regions; the alignment is coherent over the scale of a few cells, 2) there may be areas with weak diverging flows between the aligned cells, and 3 ) the horizontal (east-west) size of the cells decreases towards higher latitudes.

Lisle et al. (2004) found observational evidence of the alignment of supergranular cells in low latitude regions below 60 degrees, and suggested that the alignment is controlled by giant cells, because converging flows at the giant cell boundaries are expected to align parallel to the rotation axis at low latitudes, as predicted by numerical simulations (Brun \& Toomre 2002; Miesch et al. 2006). The alignment reported by Lisle et al. (2004) was found during 8-day observations, while our observing runs were only 16 hours long. Since the lifetime of a supergranular cell is about a day, the alignment found by them is somewhat statistical, i.e., it does not mean that supergranules are aligned at a given time, as was found by our study. Yet, in both cases the alignment may be a result of giant-cell flows organizing smaller-scale flow fields. What we essentially need for further study is to determine the lifetime of the organized structures, and to compare with the expected giant-cell lifetime (about a month), as well as to study the relationship with the global magnetic structure of the Sun. Also, it should be interesting to compare the polar supergranulation pattern with the global supergranulation pattern by using the latest helioseismology instrument, Helioseismic and Magnetic Imager (HMI) onboard the Solar Dynamics Observatory.

Perhaps, the fact that the cells in the polar regions seem to have areas with weak diverging flows between the aligned cells can be explained by the convective rolls. If there are elongated cells in the north-south direction with counter-rotating convective motions, the outward-inward travel-time difference maps would show these patterns. However, the theory (e.g. Busse 1970) predicts the convective roll patterns in low-latitude regions of the convection zone below the surface, which remain to be detected.

Hinode is a Japanese mission developed and launched by ISAS/JAXA, with NAOJ as domestic partner and NASA and STFC (UK) as international partners. It is operated by these agencies in co-operation with ESA and NSC (Norway). This work was partly carried out at the NAOJ Hinode Science Center, which is supported by the Grantin-Aid for Creative Scientific Research "The Basic Study 
of Space Weather Prediction" from MEXT, Japan (Head Investigator: K. Shibata), generous donations from Sun Microsystems, and NAOJ internal funding. Part of this work was done while K. Nagashima had been supported by the Research Fellowship from the Japan Society for the Promotion of Science for Young Scientists. This research was supported by NASA grants NNX09AB10G and NNX09AG81G.

Facility: Hinode (SOT)

\section{REFERENCES}

Bogart, R. S., Sá, L. A. D., Duvall, T. L., Haber, D. A., Toomre, J., \& Hill, F. 1995, in ESA Special Publication, Vol. 376, Helioseismology, 147-150

Brun, A. S., \& Toomre, J. 2002, ApJ, 570, 865

Brune, R., \& Wöhl, H. 1982, Sol. Phys., 75, 75

Busse, F. H. 1970, Journal of Fluid Mechanics, 44, 441

De Rosa, M. L., Gilman, P. A., \& Toomre, J. 2002, ApJ, 581, 1356

Dikpati, M., Gilman, P. A., de Toma, G., \& Ulrich, R. K. 2010, Geophys. Res. Lett., 37, 14107

Duvall, Jr., T. L., et al. 1997, Sol. Phys., 170, 63

Gilman, P. A. 1979, ApJ, 231, 284

Gizon, L., Duvall, T. L., \& Schou, J. 2003, Nature, 421, 43

Green, C. A., \& Kosovichev, A. G. 2007, ApJ, 665, L75

Hart, A. B. 1954, MNRAS, 114, 17

Hirzberger, J., Gizon, L., Solanki, S. K., \& Duvall, T. L. 2008, Sol. Phys., 251, 417

Kosovichev, A. G., \& Duvall, Jr., T. L. 1997, in Astrophysics and Space Science Library, Vol. 225, SCORe'96 : Solar Convection and Oscillations and their Relationship, ed. F. P. Pijpers, J. Christensen-Dalsgaard, \& C. S. Rosenthal (Dordrecht: Kluwer), 241

Kosugi, T., et al. 2007, Sol. Phys., 243, 3

Leighton, R. B., Noyes, R. W., \& Simon, G. W. 1962, ApJ, 135, 474
Lisle, J. P., Rast, M. P., \& Toomre, J. 2004, ApJ, 608, 1167

Miesch, M. S., Brun, A. S., \& Toomre, J. 2006, ApJ, 641, 618

Münzer, H., Hanslmeier, A., Schröter, E. H., \& Wöhl, H. 1989, A\&A, 213,431

Rast, M. P., Lisle, J. P., \& Toomre, J. 2004, ApJ, 608, 1156

Rieutord, M., \& Rincon, F. 2010, Living Reviews in Solar Physics, 7,2

Schou, J. 2003, ApJ, 596, L259

Sekii, T., et al. 2007, PASJ, 59, S637

Shimizu, T., et al. 2008, Sol. Phys., 249, 221

Simon, G. W., \& Leighton, R. B. 1964, ApJ, 140, 1120

Snodgrass, H. B. 1984, Sol. Phys., 94, 13

Stein, R. F., Benson, D., Georgobiani, D., Nordlund, A., \& Schaffenberger, W. 2007, in American Institute of Physics Conference Series, Vol. 948, Unsolved Problems in Stellar Physics:

A Conference in Honor of Douglas Gough, ed. R. J. Stancliffe, G. Houdek, R. G. Martin, \& C. A. Tout, 111-115

Tsuneta, S., et al. 2008, Sol. Phys., 249, 167

Woodard, M. F. 2007, ApJ, 668, 1189

Ye, B., \& Livingston, W. 1998, Sol. Phys., 179, 1

Zaatri, A., Corbard, T., Roth, M., González Hernández, I., \& von der Lühe, O. 2008, Journal of Physics Conference Series, 118, 012090 
(a)

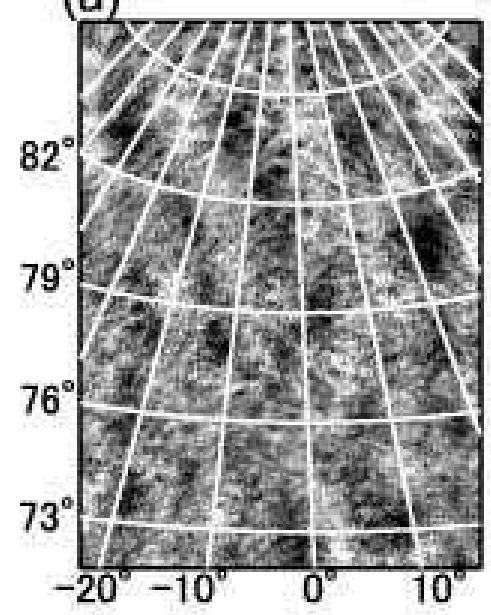

(b)

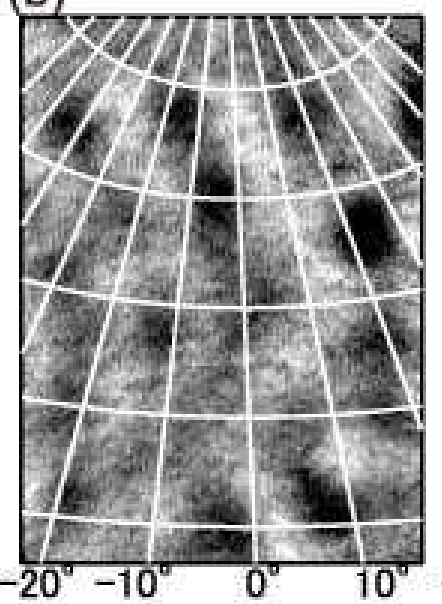

(c)

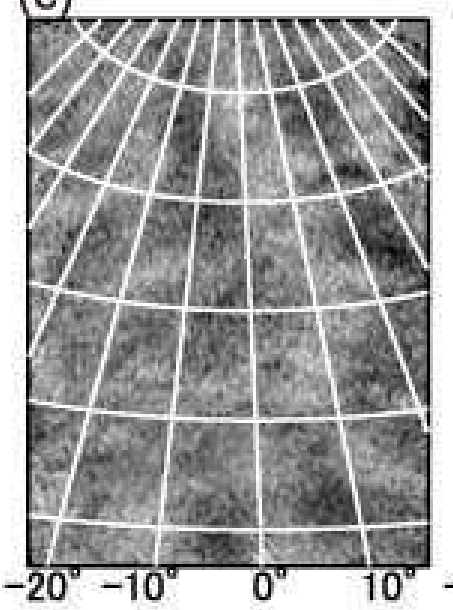

(d)

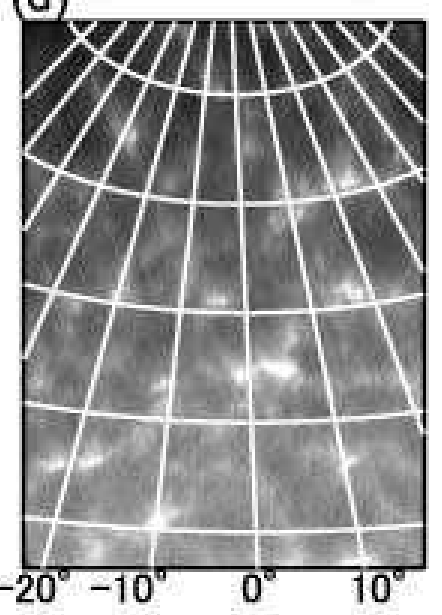

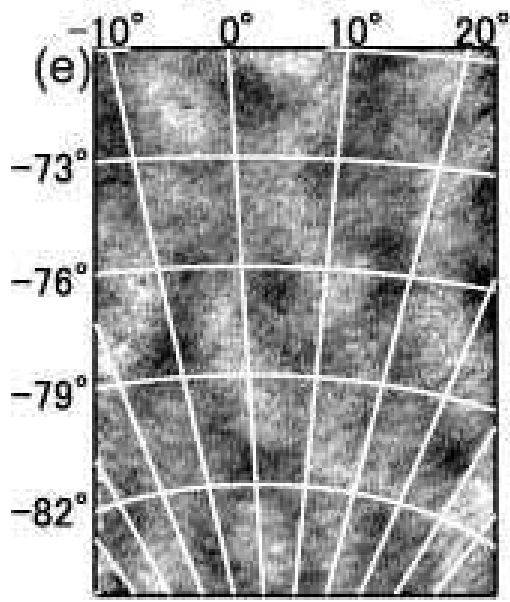
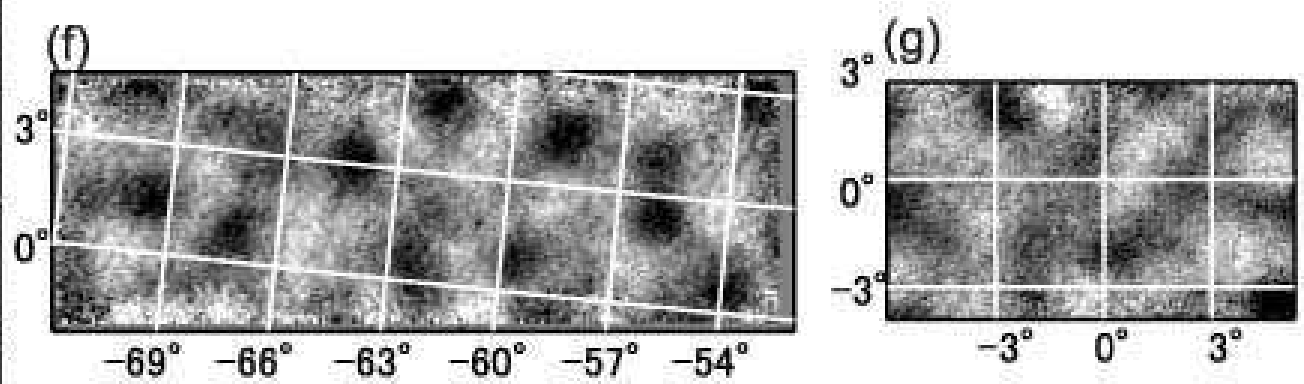

FIG. 1. - Outward-inward travel-time difference maps of the north polar region on 2009 September 25, for travel distances: (a) 8.4 Mm, (b) $14.4 \mathrm{Mm}$, and (c) $21.1 \mathrm{Mm}$. Panel (d) shows a 4-hour averaged intensity image in the Ca II H line of the region. Panels (e), (f), and (g) show the outward-inward travel-time difference maps of the south polar region on 2009 March 7, near the east limb on 2009 September 27, and around the disk center on 2009 December 11, for the travel distances of $14.4 \mathrm{Mm}$. The gray scale used for the travel-time difference maps covers the range from -0.5 minutes (black) to +0.5 minutes (white). 

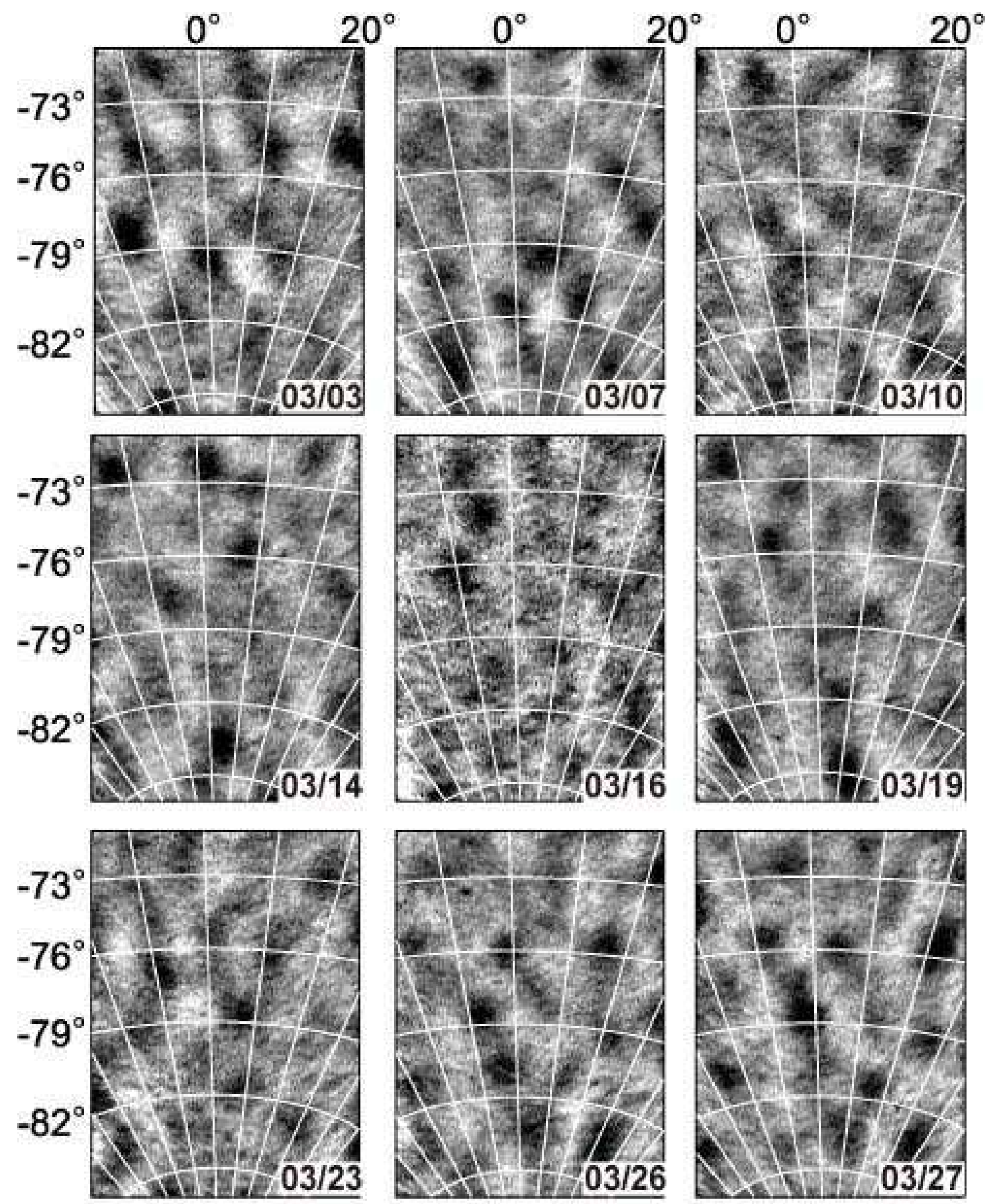

FIG. 2.- Outward-inward travel-time difference maps of the south polar region observed for 9 periods in March of 2010. The observing dates are indicated in the lower right corner. Note that the March 16 map is noisier than others because of short observation period. The gray scale is same as in Figure 1 

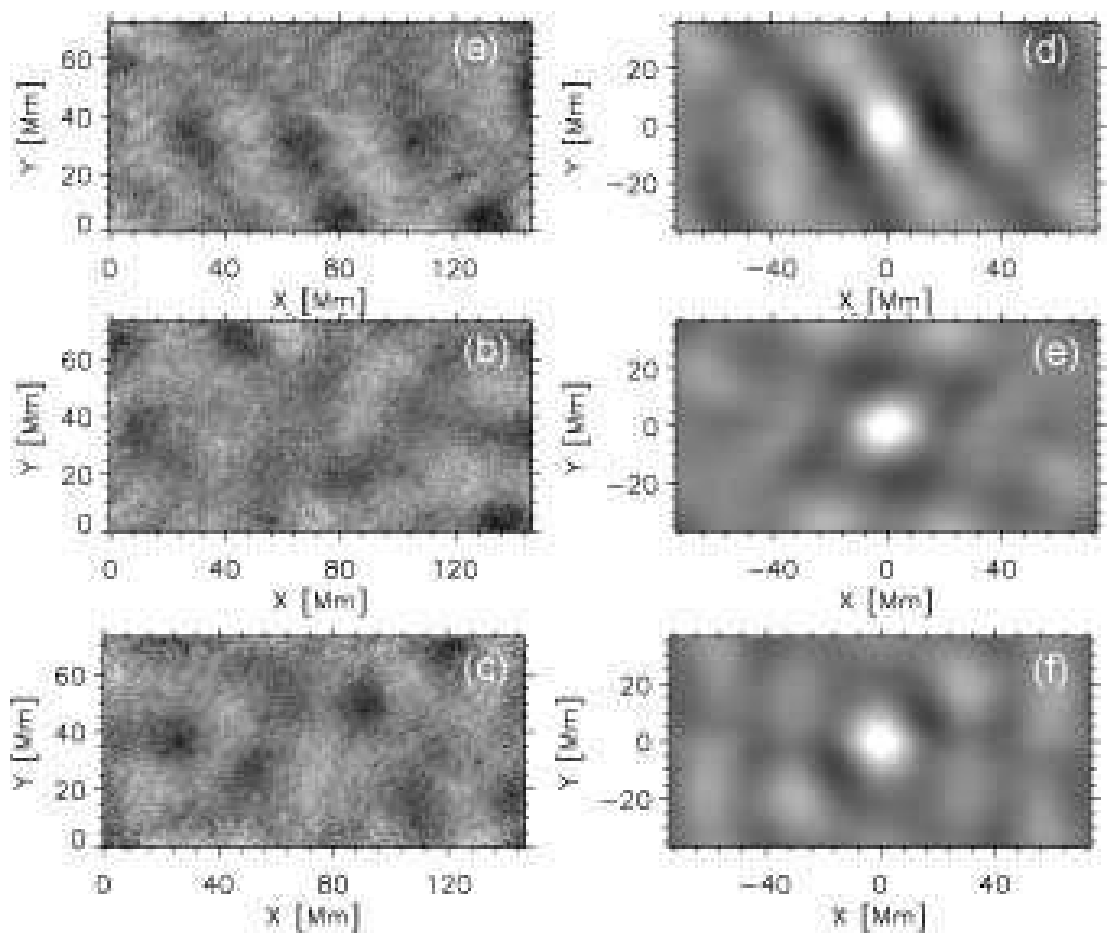

Fig. 3. - Travel-time difference maps and two-dimensional correlation maps of the travel-time difference. Left column: Travel-time difference maps in (a) the north polar region, (b) the disk center region, and (c) the east limb region. These maps are Postel's projected maps, and the sizes of the field-of-view are the same. Note that (a) and (c) is only a part of the full field of view. The gray scale is same as in Figure 1 Right column: Two-dimensional correlation maps of the travel-time difference maps shown in the left panels. Panels (d), (e), and (f) are for the north polar region, the disk center region, and the east limb region, respectively. The gray-scale range of the cross-correlation function is from -0.5 (black) to +0.5 (white).
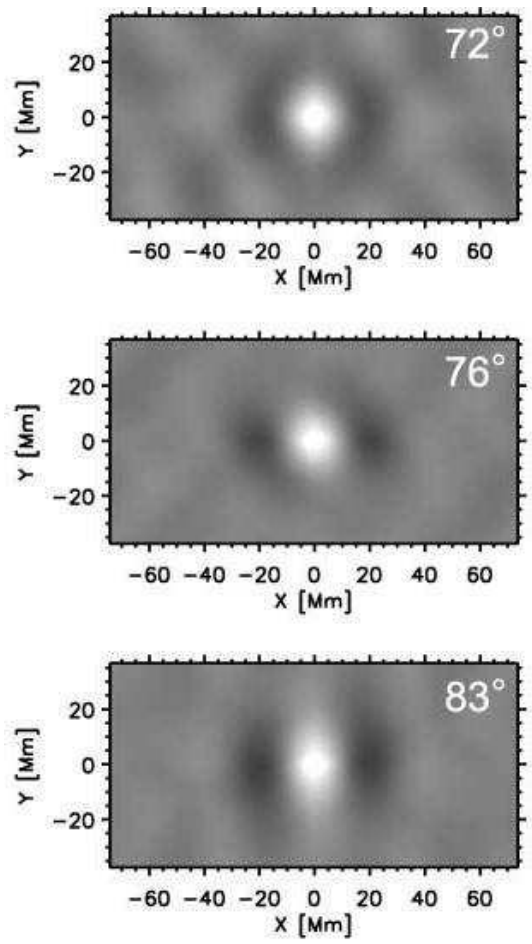

FIG. 4.- Averages of the two-dimensional cross-correlation functions over all the nine south pole datasets for the central latitudes: $72^{\circ}$ (top), $76^{\circ}$ (middle), and $83^{\circ}$ (bottom). The gray scale is same as in the right panels of Figure 3 


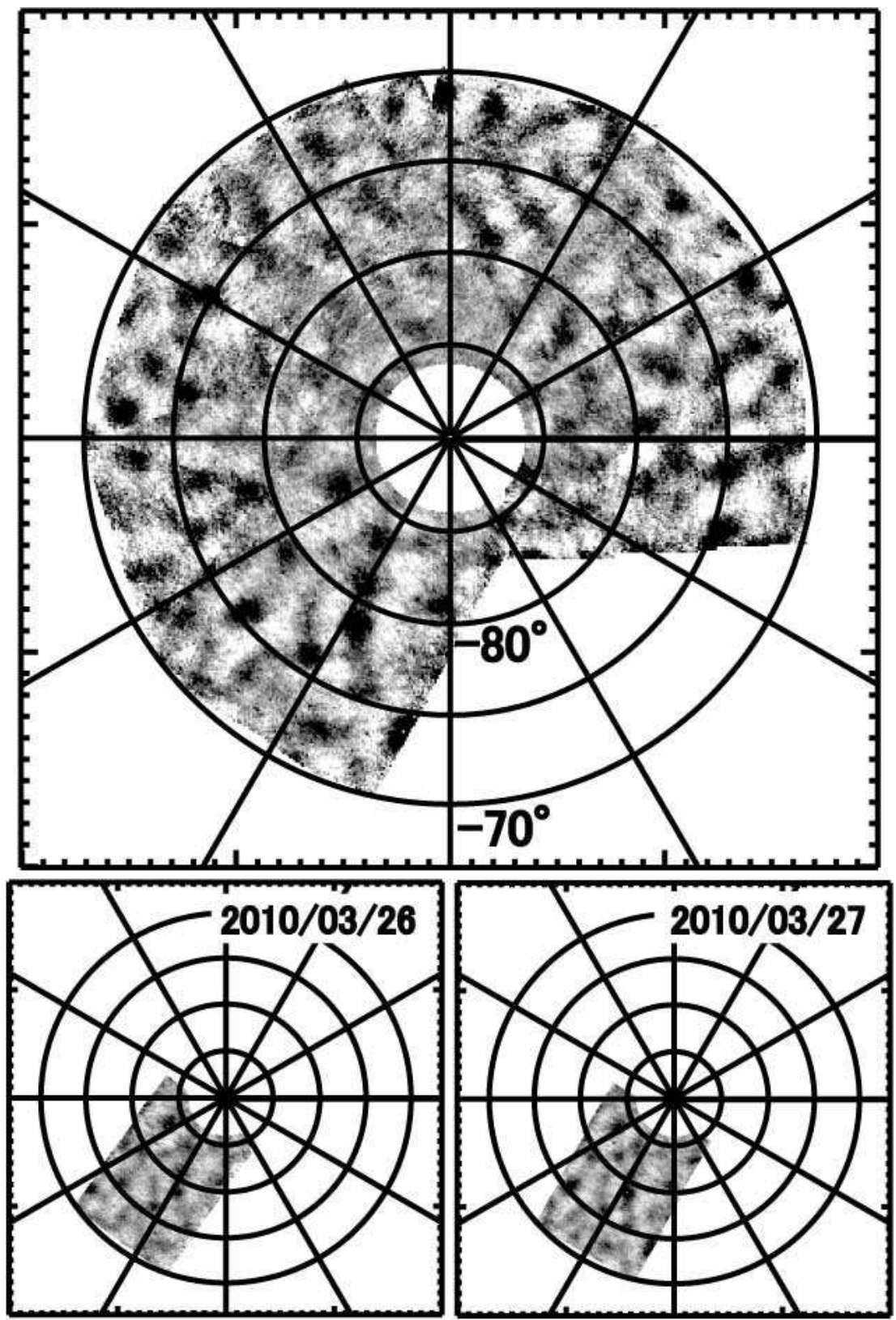

FIG. 5.- Outward-inward travel-time difference maps of the south polar region observed in March of 2010. The upper panel shows the combined polar view above the South Pole, obtained by overlapping the results of the nine observing runs. The lower panels are the last two consecutive travel-time difference maps. The concentric circles are the latitudinal lines spaced by five degrees, while the straight lines are the longitudinal lines spaced by 30 degrees. The gray scales used for the upper panel and the lower panels cover the range from -0.25 minutes to +0.25 minutes and the range from -0.5 minutes to +0.5 minutes, respectively. 